\title{
Transition from turbulent to nearly laminar vortex flow in superconductors with periodic pinning
}

\author{
J. Gutierrez, A. V. Silhanek, J. Van de Vondel, W. Gillijns, and V. V. Moshchalkov \\ Institute for Nanoscale Physics and Chemistry (INPAC), Nanoscale Superconductivity and Magnetism Group, \\ K.U. Leuven, Celestijnenlaan 200D, B-3001 Leuven, Belgium
}

(Received 8 September 2009; published 28 October 2009)

\begin{abstract}
We revisit the vortex dynamics in Al thin films containing an artificial periodic array of antidots by means of electrical transport measurements. We clearly identify a turbulent to laminarlike vortex flow transition which manifests itself as a negative differential resistivity. This transition is accompanied by a strong irreversibility in the voltage-current characteristics. The dynamical phase diagrams obtained as a function of commensurability, temperature, and driving force are in good agreement with the early predictions by Reichhardt et al. [Phys. Rev. Lett. 78, 2648 (1997)] based on molecular dynamic simulations.
\end{abstract}

DOI: $10.1103 /$ PhysRevB.80.140514

PACS number(s): 74.25.Qt, 74.25.Dw, 74.40.+k, 74.78.Na

The pioneering work of Giaever ${ }^{1}$ provided the first experimental evidence that dc electrical resistance in type-II superconductors is a direct consequence of the motion of Abrikosov vortices.

Extensive molecular dynamics (MD) simulations of array of vortices treated as overdamped objects interacting repulsively with each other and moving over a predefined potential landscape have proven to be a remarkably successful tool to describe the vortex dynamics in all sort of superconductors. ${ }^{2}$ Superconductors with a periodic array of pinning centers represent a unique playground to investigate the interaction between vortices and pinning sites. ${ }^{3-5}$ Early MD simulations for this particular system by Reichhardt $e t$ $a l .6,7$ predicted a remarkable property, namely, the existence of a dynamic transition from a highly dissipative disordered vortex flow at low driving forces to a less dissipative state of ordered flow where pinned vortices coexist with fast solitonlike excitations at higher currents. The sequence in which these dynamical phases appear is highly nontrivial and even counterintuitive since it implies a reduction of the average vortex mobility by increasing the driving force.

In spite of the great deal of attention that this fascinating discovery has received in the superconducting community, so far there has been no experimental confirmation of it. A possible cause, recently pointed out by Misko et al., ${ }^{8,9}$ is that strong heating or disorder effects can mask this effect. In this Rapid Communication we provide unambiguous experimental evidence that a superconducting film with a periodic array of antidots exhibits a clear turbulent to laminarlike vortex flow transition provided that heat removal is efficient. This transition appears as a negative differential resistivity in the current-voltage characteristics. We demonstrate that the loci of the dynamic transitions in a magnetic field-temperature phase diagram are highly sensitive to the pinning strength and commensurability of the system. At high bias currents the simplified model of a vortex as a pointlike classical particle which ignores the internal structure of the vortex and its elastic nature seems to fail and more realistic approaches, such as time dependent Ginzburg-Landau formalism, become necessary.

The investigated sample consists of an $\mathrm{Al}$ film of thickness $t=50 \mathrm{~nm}$ grown by electron beam evaporation on top of a $\mathrm{SiO}_{2}$ substrate with a predefined resist mask. The $\mathrm{Al}$ film is patterned in a 2-mm-long and $600-\mu \mathrm{m}$-wide bridge. The whole Al film contains a periodic array of artificial pinning centers (APCs) which consists of a square lattice of square antidots, with lattice parameter $d=3 \mu \mathrm{m}$ and antidot size $a$ $=0.6 \mu \mathrm{m}$. The electrical transport measurements were carried out in a four-probe geometry in a ${ }^{4} \mathrm{He}$ bath cryostat with a base temperature of $1.1 \mathrm{~K}$ and temperature stability better than $100 \mu \mathrm{K}$. The normal/superconductor transition temperature $\left(T_{c}\right)$ defined at $50 \%$ of the resistivity drop is $1.313 \mathrm{~K}$ with a transition width of $\sim 6 \mathrm{mK}$. From the normal state resistivity $\rho_{0}=1.7 \times 10^{-8} \Omega \mathrm{m}$ and the slope of the upper critical field $d H_{c 2} / d T=11.86 \mathrm{mT} / \mathrm{K}$ and using the expressions for the penetration depth and coherence length for amorphous superconductors described in Ref. 11 we find $\Lambda(0)=\lambda^{2}(0) / t=288 \mathrm{~nm}$ and $\xi(0)=145 \mathrm{~nm}$.

The first matching field at which the density of vortex lines equals the density of pinning centers is $B_{1}=\Phi_{0} / d^{2}$ $=0.23 \mathrm{mT}$, where $\Phi_{0}=2.07 \times 10^{-15} \mathrm{Tm}^{2}$ is the flux quanta. The inset of Fig. 1 shows that these commensurability effects lead to a sharp increase of the magnetoresistance at $B=B_{1}$ indicating that a maximum of one flux quanta per antidot can be trapped. This result is consistent with the fact that the condition ${ }^{10} a^{3}>\pi^{3 / 2} \xi \lambda^{2}$, needed for the trapping of two flux quanta per site, is only satisfied at temperatures below 0.95 $\mathrm{K}$.

Under these circumstances, magnetic fields slightly exceeding the first matching field $B_{1}$ ensure the presence of two different kinds of vortices in the system. Vortices sitting in the APCs, which are strongly pinned, and interstitial vortices weakly pinned by the caging potential produced by the neighboring trapped vortices. ${ }^{4,5}$ It is expected that by increasing the external drive, first the interstitial vortices will be set in motion whereas those in the antidots remain immobile $^{5}$ as depicted in Fig. 1(b). In order to identify these regimes of the vortex motion we measured the electric fieldcurrent density characteristics $(E-J)$ at different temperatures and fields. It is important to mention that every $E-J$ characteristic is obtained after a field cooling procedure, thus ensuring to start from a vortex configuration close to the equilibrium. Figure 1 shows a semilogarithmic plot of a $E-J$ curve for $T=1.278 \mathrm{~K}$ and $B=1.031 B_{1}$. 


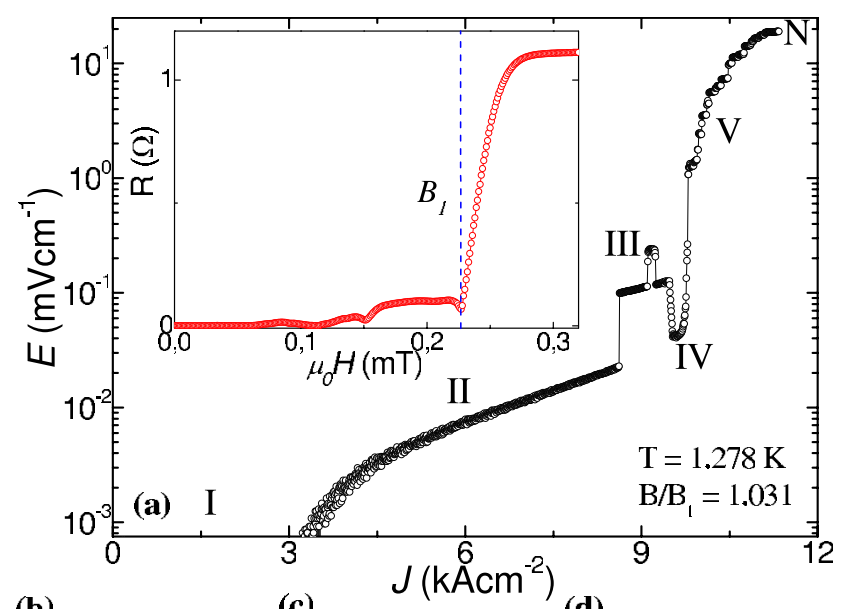

(b) (c)
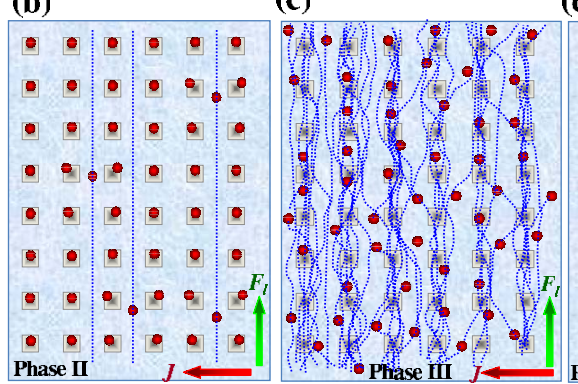

(d)

FIG. 1. (Color online) (a) $E-J$ curve taken at $T=1.278 \mathrm{~K}$ and for $B / B_{1}=1.036$. Dynamical vortex phases $\mathrm{I}$-pinned lattice, II-1D interstitial vortex motion, III-2D disordered motion of the flux line lattice, IV_-1D incommensurate motion, V-phase slip lines formation, and $\mathrm{N}$-normal state. The inset shows the resistance versus the applied magnetic field at $1.295 \mathrm{~K}$. The drop in $R$ is observed exactly at $B_{1}$. Sketches of the interstitial vortex motion in (b) phase II of the turbulentlike 2D motion of vortices in (c) phase III and of the 1D incommensurate row motion in (d) phase IV.

Six different dynamic regimes, labeled $\mathrm{I}-\mathrm{V}$ and normal state $(\mathrm{N})$, can be observed. At low currents a dissipationless state indicates the absence of vortex motion (region I). Above a certain threshold value $\left(J \sim 3.4 \mathrm{kA} \mathrm{cm}^{-2}\right)$ an electric field appears due to the one-dimensional (1D) motion of weakly pinned interstitial vortices along the rows of strongly pinned ones ${ }^{6}$ (region II). Further increasing $J$ leads to an abrupt jump of $E$ (region III) followed by a sudden reduction of the dissipation around $J=9.6 \mathrm{kA} \mathrm{cm}^{-2}$ (region IV). Notice that this last transition is counterintuitive since it implies the decrease of average vortex mobility even though the external drive is increased.

Molecular dynamics simulations performed by Reichhardt et $a l^{6}{ }^{6}$ actually predicted these phases. According to these authors, phase III consists of a disordered (turbulentlike) two-dimensional (2D) in plane motion involving all the flux $\operatorname{lines}^{7}$ as schematically shown in Fig. 1(c). The physical mechanism causing the sudden first-order-like transition from II to III is an increase of the repulsive transversal force (i.e., perpendicular to the Lorentz force) acting on the pinned vortices as a result of the flow of interstitial vortices. As $J$ increases, eventually the perpendicular force is enough to depin the vortices sitting in the antidots. This triggers an avalanche process which rapidly increases the number of moving vortices and therefore also the electric field. MD simulations predicted strong voltage fluctuations on this phase due to the disordered motion of vortices. On the contrary our experimental observations present nearly no voltage noise. This is explained by the large measurement time (compared to the vortex motion time) and the size of the experimental system, which tends to average out the voltage signal. As the driving force increases, the component of the velocity parallel to the APC rows $v_{L}$ increases whereas the distribution of transverse velocities $v_{T}$ remains unaltered. For a high enough $J$ the time of flight of a vortex to move from antidot to antidot $\left(\sim d / v_{L}\right)$ is shorter than the time needed to escape from a moving vortex row $\left(\sim a / v_{T}\right)$ resulting in a dynamic confinement of the vortex lattice. In this new phase (region IV), two types of vortex rows coexist, those with an excess of vortices (incommensurate) and others with the same number of vortices than antidots (commensurate). The commensurate rows remain nearly pinned and therefore the electric field is solely generated by the 1D movement of the incommensurate rows leading to the abrupt decrease of the dissipation, ${ }^{7}$ as depicted in Fig. 1(d).

Phase IV takes place in a narrow current range before entering in a new regime $\mathrm{V}$. This is markedly different from MD predictions. The reason for this discrepancy is that in $\mathrm{Al}$, nonequilibrium processes (ignored in molecular dynamics simulations) start at relatively low vortex velocities due to the large electron-phonon and electron-electron relaxation times. The proliferation of nonequilibrium effects will shorten the region where phase IV appears and it can even hinder the visualization of the negative differential effect. Phase $\mathrm{V}$ is marked by the sequential appearance of jumps in $E$ followed by plateaus. This behavior is characteristic of phase-slip centers and has been extensively explored in the past. ${ }^{12,13,16}$ Most likely, in our particular case, the steps in $E$ coincide with the generation of phase-slip lines at the incommensurate moving vortex rows where vortices have a higher average mobility. ${ }^{14}$ Interestingly, these phase-slip lines may coexist with slow moving vortex rows (commensurate rows). For the field and temperature configuration present in Fig. 1, the phase-slip region $(\mathrm{V})$ eventually ends up in the normal state region $(\mathrm{N})$ at about $11 \mathrm{kA} \mathrm{cm}^{-2}$. Clearly, both regimes, $\mathrm{V}$ and $\mathrm{N}$, cannot be properly described by molecular dynamics approximations and the incorporation of the GinzburgLandau formalism is needed. ${ }^{14,15}$

In order to investigate the region of stability of all these regimes we have performed measurements at different magnetic fields and temperatures. Figure 2 summarizes the different possible responses obtained in our particular system. The $E-J$ curve represented by open (black) circles in panel (a) of Fig. 2 has been measured by ramping up the current. All the different phases described in Fig. 1(a) are still observed. The $E-J$ curve defined by open (red) stars has been measured by ramping down the current to zero starting from the end of phase IV $\left(\sim 10 \mathrm{kA} \mathrm{cm}^{-2}\right)$. A pronounced irreversibility in the III-IV and II-III boundaries is observed. When ramping down the current, phases III and IV [marked in italic in Fig. 2(a)] are stable for a wider current range. Particularly the disordered phase III shows a strong irreversibility, remaining stable down to very low currents, jumping down to phase II close to the I-II boundary. Within the MD 


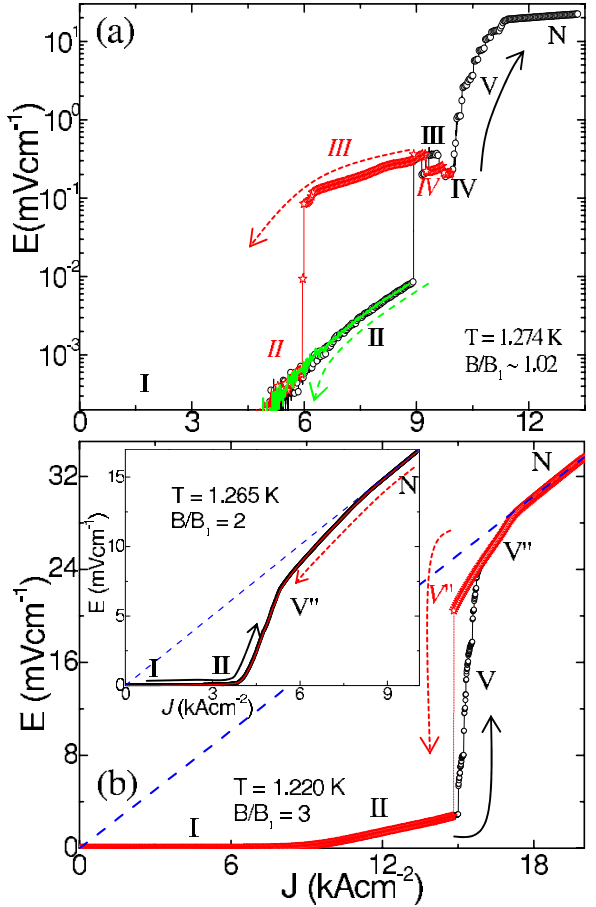

FIG. 2. (Color online) $E-J$ curves ramping up and down the driving current taken at (a) $T=1.274 \mathrm{~K}, B / B_{1}=1.01$ and (b) $T$ $=1.220 \mathrm{~K}, B / B_{1}=3$. Inset shows a fully reversible $E-J$ curve taken at $T=1.265 \mathrm{~K}, B / B_{1}=2$. When a irreversibility is present phases are labeled in italic for the ramping down case. Dashed blue lines are fittings to the Ohmic behavior in the normal state.

framework, irreversibilities in the II-III and III-IV transitions were also predicted, suggesting that these changes of regime exhibit a truly first-order-like character. ${ }^{7}$ In other words, when crossing the II-III boundary ramping up the driving force, only interstitial vortices move, whereas reversing the crossing from II to III by ramping down the current implies, for the same applied current, a disordered flow involving all vortices and therefore higher dissipation. This interpretation is confirmed by the fact that as long as the maximum current lies within phase II, no irreversibilities are observed, as shown by the solid (green) line in Fig. 2(a).

It is worth noticing that both phases III and IV are very sensitive to the temperature and magnetic field. As it is shown in Fig. 2(b), by detuning the field far from the first matching condition or decreasing the temperature, these dynamic regimes disappear. In addition, we observe that the discrete jumps in $E$ produced by phase-slip lines (phase V) give way to a smooth increase of $E$ with $J$ (phase $\mathrm{V}^{\prime \prime}$ ) before ending into the normal state [ramping up $E-J$ curves represented by open (black) circles in Figs. 2(b)]. Remarkably, this smooth increase of dissipation exhibits no irreversibility at all as observed in the inset of Fig. 2(b). This behavior is symptomatic of an efficient heat removal in the present system and gives confidence to the interpretation of the low current data, based on simple MD simulations.

In Fig. 3 we summarize the evolution of the different dynamic regimes as a function of field (a) and temperature (b). We have limited our measurements to the region where phase III (negative differential resistivity) appears. Increas-

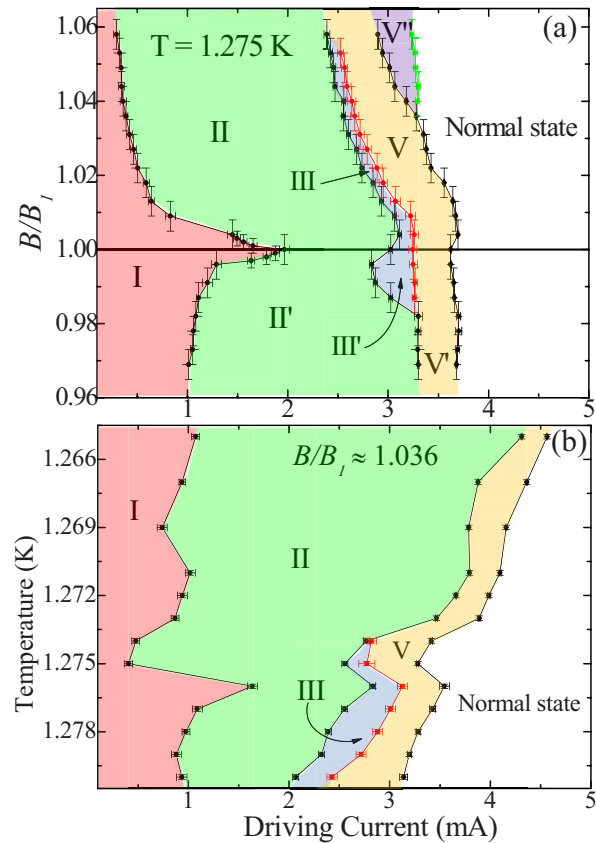

FIG. 3. (Color online) Dynamic phase diagrams as function of (a) the commensurability and the driving current taken at $T$ $=1.275 \mathrm{~K}$ and $(\mathrm{b})$ the temperature and the driving current for $B / B_{1}=1.036$.

ing further the magnetic field produces a fast development of phase $\mathrm{V}^{\prime \prime}$ which finally meets phase II engulfing the other phases. For the sake of clarity phase IV has not been included due to its short extend in current. In this diagram we have labeled the phases below $B_{1}$ with an accent to differentiate them from phases above $B_{1}$. This distinction emphasize the fact that above $B_{1}$ the vortex dynamics is given by the contribution of trapped vortices and interstitial vortices, whereas below $B_{1}$ the response of the system is dominated by the motion of vortex vacancies in the rows of antidots. ${ }^{7}$

As it is observed in this phase diagram, a significantly higher driving force is needed to start the motion of vacancies $\left(\mathrm{II}^{\prime}\right)$ as compared to interstitial vortices (II). An interesting point of this diagram is the sharp increase in the current needed to transit from phase I to phase II at $B_{1}$. It is expected that when the number of vortices levels the number of antidots the dissipation will set in when the driving force is high enough to depin the entire vortex lattice. Based on this consideration, a transition from phase I to $\mathrm{N}$ would be expected. ${ }^{6}$ In a real sample this condition is hard to achieve due to unavoidable imperfections in the pinning array or even due to the inhomogeneous field distribution due to the driving current.

In Fig. 3(b) we have plotted the evolution of the dynamic vortex phase diagram as a function of temperature and driving current. Since the pinning force decreases monotonously with increasing temperature, this plot has a clear correspondence with Fig. 3(a) in Ref. 6. To emphasize this correspondence the vertical scale in Fig. 3(b) has been inverted. It can be seen that the disordered flow of vortices, phase III, only exists at high enough temperatures (low pinning force). This trend, which is opposite to that predicted in Ref. 6, results from the fact that the experimental depinning current ap- 
proaches the depairing current. This behavior cannot be foreseen within MD simulations since in this modeling the depairing current is considered as infinite. It is important to mention that similar investigation performed in a sample with larger antidot size but the same periodicity of the array shows no hint of phases III and IV. This finding, together with the fact that these regimes disappear at low temperature and field detuning as small as $15 \%$ away from matching condition, indicates that the negative differential resistance is very sensitive to the parameters of the pinning landscape and the thermodynamic conditions.

In conclusion, we have provided clear experimental evidence of the presence of a negative differential resistance dynamical phase in superconductors with a periodic pinning array, earlier predicted by molecular dynamic simulations. ${ }^{6}$ We have also revealed a rich variety of dynamic vortex phases and studied their stability by hysteretic $E-J$ measurements. The different phases have been mapped into dynamic phase diagrams which show good agreement with MD simulations. Our findings strongly support these previous theoretical works and we believe it will reopen the enthusiasm to study experimentally in more detail the different properties of the dynamic vortex phases. Moreover, in exploding the analogies of a vortex lattice in a periodic pinning potential with other elastic systems, the present findings can be relevant for the research on dynamic phases in Wigner crystals $^{17,18}$ and colloidal molecular crystals pinned by an array of optical traps. ${ }^{19,20}$

This work was supported by Methusalem Funding by the Flemish Government, the FWO-Vlaanderen, and the Belgian Inter-University Attraction Poles IAP Programmes. J.G. is grateful for the financial support of the Spanish Ministerio de Ciencia e Innovacion. A.V.S., J.V.d.V., and W.G. are grateful for the support from the FWO-Vlaanderen. The authors want to give thanks to D. Vodolazov for useful discussions.
${ }^{1}$ I. Giaever, Phys. Rev. Lett. 15, 825 (1965).

${ }^{2}$ E. H. Brandt, Phys. Rev. Lett. 50, 1599 (1983).

${ }^{3}$ A. T. Fiory, A. F. Hebard, and S. Somekh, Appl. Phys. Lett. 32, 73 (1978).

${ }^{4}$ M. Baert, V. V. Metlushko, R. Jonckheere, V. V. Moshchalkov, and Y. Bruynseraede, Phys. Rev. Lett. 74, 3269 (1995).

${ }^{5}$ E. Rosseel, M. Van Bael, M. Baert, R. Jonckheere, V. V. Moshchalkov, and Y. Bruynseraede, Phys. Rev. B 53, R2983 (1996).

${ }^{6}$ C. Reichhardt, C. J. Olson, and F. Nori, Phys. Rev. Lett. 78, 2648 (1997).

${ }^{7}$ C. Reichhardt, C. J. Olson, and F. Nori, Phys. Rev. B 58, 6534 (1998).

${ }^{8}$ V. R. Misko, S. Savel'ev, A. L. Rakhmanov, and F. Nori, Phys. Rev. Lett. 96, 127004 (2006).

${ }^{9}$ V. R. Misko, S. Savel'ev, A. L. Rakhmanov, and F. Nori, Phys. Rev. B 75, 024509 (2007).

${ }^{10}$ A. I. Buzdin, Phys. Rev. B 47, 11416 (1993).

${ }^{11}$ P. H. Kes and C. C. Tsuei, Phys. Rev. B 28, 5126 (1983), see Eqs. (A10) and (A13).
${ }^{12}$ J. D. Meyer, Appl. Phys. (Berlin) 2, 303 (1973).

${ }^{13}$ W. Skocpol, M. R. Beasley, and M. Tinkham, J. Low Temp. Phys. 16, 145 (1974).

${ }^{14}$ D. Y. Vodolazov and F. M. Peeters, Phys. Rev. B 76, 014521 (2007).

${ }^{15}$ G. R. Berdiyorov, M. V. Milosevic, and F. M. Peeters, Phys. Rev. B 79, 184506 (2009); G. R. Berdiyorov, A. K. Elmurodov, F. M. Peeters, and D. Y. Vodolazov, ibid. 79, 174506 (2009).

${ }^{16}$ A. G. Sivakov, A. M. Glukhov, A. N. Omelyanchouk, Y. Koval, P. Muller, and A. V. Ustinov, Phys. Rev. Lett. 91, 267001 (2003).

${ }^{17}$ G. Coupier, M. Saint Jean, and C. Guthmann, Phys. Rev. B 75, 224103 (2007).

${ }^{18}$ V. J. Goldman, M. Santos, M. Shayegan, and J. E. Cunningham, Phys. Rev. Lett. 65, 2189 (1990).

${ }^{19}$ C. Reichhardt and C. J. Olson, Phys. Rev. Lett. 88, 248301 (2002).

${ }^{20}$ M. Brunner and C. Bechinger, Phys. Rev. Lett. 88, 248302 (2002). 